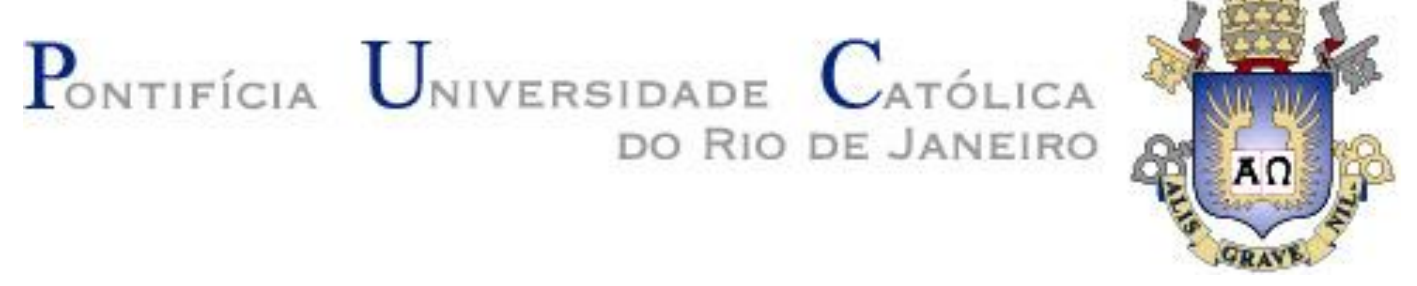

Raquel Jauffret Guilhon

Geração automática de suíte de teste para GUI a partir de Rede de Petri

Dissertação de Mestrado

Dissertação apresentada como requisito parcial para obtenção do grau de Mestre pelo Programa de Pós-graduação em Informática da PUC-Rio.

Orientador: Prof. Arndt Von Staa 




Raquel Jauffret Guilhon

\section{Geração automática de suíte de teste para GUI a partir de} Rede de Petri

Dissertação apresentada como requisito parcial para obtenção do grau de Mestre pelo Programa de Pós-graduação em Informática da PUC-Rio. Aprovada pela Comissão Examinadora abaixo assinada.

Prof. Arndt Von Staa

Orientador

Departamento de Informática - PUC-Rio

Prof. Alberto Barbosa Raposo

Departamento de Informática - PUC-Rio

Prof. Hélio Cortes Vieira Lopes

Departamento de Informática - PUC-Rio

Prof. José Eugênio Leal Coordenador Setorial do Centro Técnico Científico - PUC-Rio

Rio de Janeiro, 22 de Agosto de 2014 
Todos os direitos reservados. É proibida a reprodução total ou parcial do trabalho sem autorização da universidade, da autora e do orientador.

\section{Raquel Jauffret Guilhon}

Graduou-se em Engenharia da Computação pela Pontifícia Universidade Católica do Rio de Janeiro, em 2009. Atua como Analista de Sistemas em um projeto de pesquisa do instituto Tecgraf/PUC-Rio desde 2009. Interessada em prover soluções práticas e funcionais para problemas de desenvolvimento de software em geral.

Ficha Catalográfica

Guilhon, Raquel Jauffret

Geração automática de suíte de teste para GUI a partir de Rede de Petri / Raquel Jauffret Guilhon ; orientador: Arndt Von Staa. - 2014.

110 f. : il. (color.) ; $30 \mathrm{~cm}$

Dissertação(mestrado)-Pontifícia Universidade Católica do Rio de Janeiro, Departamento de Informática, 2014.

Inclui bibliografia

1. Informática - Teses. 2. Rede de Petri. 3. Teste de GUI. 4. Teste baseado em modelo. I. Staa, Arndt Von. II. Pontifícia Universidade Católica do Rio de Janeiro. Departamento de Informática. III. Título. 


\section{Agradecimentos}

À minha família, pelo apoio, respeito e amor de sempre.

A meu orientador Arndt Von Staa, pela colaboração fundamental para a realização deste trabalho e pela forma como conduziu a orientação.

Ao Pedro Mário Cruz e Silva, coordenador do projeto V3O2/Tecgraf, por me conceder a oportunidade deste mestrado e pelo apoio ao longo da etapa.

A todos os amigos do $\mathrm{V} 3 \mathrm{O} 2$, pelo entendimento, carinho e apoio de sempre.

Aos professores da banca examinadora, por terem aceitado fazer parte desta importante etapa. 


\section{Resumo}

Guilhon, Raquel Jauffret; Staa, Arndt Von. Geração automática de suíte de teste para GUI a partir de Rede de Petri. Rio de Janeiro, 2014. 110p. Dissertação de Mestrado - Departamento de Informática, Pontifícia Universidade Católica do Rio de Janeiro.

$\mathrm{Na}$ etapa de testes de software, as falhas podem ser reveladas e em seguida diagnosticadas a fim de identificar os defeitos que a causaram. Os testes devem idealmente ser aplicados desde o nível da unidade até o nível mais elevado do software, como o teste de sistema. Em um destes níveis está o teste da interface gráfica, ou Graphical User Interface (GUI). Garantir o correto funcionamento da GUI quanto ao estado de seus elementos após diversos eventos de usuário, é tão importante quanto o teste de outras camadas, pois a GUI é um meio direto de interação com a aplicação, sendo o recurso que mais influencia em como será qualificada a experiência pelo usuário final. Este trabalho propõe uma abordagem de teste baseado em modelo, ou Model-Based Testing (MBT), utilizando Redes de Petri (RP) de alto nível para representar a interface gráfica. A Rede de Petri é uma ferramenta de modelagem e uma linguagem de especificação de base matemática que define graficamente a estrutura de sistemas especialmente concorrentes. Uma característica importante das RPs é que elas podem ser simuladas, permitindo que se observe o comportamento do sistema e que se obtenha a geração de casos de teste a partir dos caminhos executados na simulação. Foi investigada a geração de suítes de teste para interface gráfica a partir do modelo Rede de Petri. Para isso, considerou-se a relação entre as ações do usuário e os estados resultantes na GUI, percebendo como uma RP pode modelá-los. Uma ferramenta de apoio foi desenvolvida para que, a partir das simulações realizadas na Rede de Petri, suítes de teste fossem geradas na linguagem $\mathrm{C}++$, tornando possível a execução automática em um software de estudo. Por fim, o critério de teste Análise de Mutantes, que mede a eficácia da suíte gerada a partir da RP, foi empregado como um dos meios de validação deste trabalho.

\section{Palavras-chave}

Rede de Petri; Teste de GUI; Teste baseado em modelo 


\section{Abstract}

Guilhon, Raquel Jauffret; Staa, Arndt Von (Advisor). GUI test suite automatic generation from Petri Net. Rio de Janeiro, 2014. 110p. MSc. Dissertation - Departamento de Informática, Pontifícia Universidade Católica do Rio de Janeiro.

In software testing stage, faults can be revealed and then diagnosed to identify defects that caused it. Tests should ideally be applied from the unit level to the higher level of software, such as system testing. In one of these levels resides GUI (Graphical User Interface) testing. Ensuring the correct operation of the GUI on the state of its elements after various user events is as important as the other layers tests, since GUI is a direct way to interact with the application, being the feature that most influences how the experience will be qualified by the end user. This paper proposes a Model-Based Testing (MBT) approach using highlevel Petri Nets (RP) to represent graphical user interface. Petri Net is a modeling tool and a mathematical specification language that graphically defines the structure of systems, specially the concurrent ones. An important feature of RPs is that they can be simulated, allowing one to observe the behavior of the system and to obtain the generation of test cases from the paths executed in the simulation. The generation of test suites for GUI from the Petri Net model was investigated. For this, we considered the relationship between user actions and resulting states in the GUI, realizing how an RP can model them. A support tool was developed so that, from the simulations of Petri Net, test suites were generated in $\mathrm{C}++$ language, making it possible to auto-run them on a study software. Finally, the Mutation Analysis test criterion, which measures the effectiveness of the suite generated from RP, was employed as a means of validation of this work.

\section{Keywords}

Petri Net; GUI Testing; Model based testing 


\section{Sumário}

1. Introdução 9

1.1 Testes de software 9

1.2 Testes em GUI 10

1.3 Motivação 14

1.4 Estrutura da Dissertação 21

2. Teste baseado em modelo 23

2.1 Redes de Petri 23

2.2 GUI como Rede de Petri 28

3. Revisão bibliográfica 36

3.1 Teste de GUI a partir de modelo 36

3.2 GUI como Rede de Petri 39

3.3 Ferramentas de teste em GUI 39

3.4 Análise de mutantes no nível funcional 40

4. Metodologia e ferramenta desenvolvida 43

4.1 Modelagem da Rede de Petri 43

4.1.1 Construção do modelo da GUI 44

4.1.2 Simulação 50

4.1.3 Exemplo de funcionalidade real 53

4.1.4 Configurações extras de modelagem 60

4.2 Geração da suíte de teste - Ferramenta guiftG 63

4.2.1 Geração da suíte de teste "genérica" 66

4.2.2 Geração da suíte especializada para uma $\quad 67$ ferramenta de execução de teste automatizada

4.3 Execução dos testes no SUT (Software Under Test) 69

5. Critério de teste Análise de Mutantes 70

5.1 AM tradicional $\quad 72$

5.2 AM no nível funcional e de sistema 73 
5.3 Análise de Mutantes no SUT 73

5.3.1 Operadores 74

5.3.2 Ferramenta desenvolvida 75

$\begin{array}{ll}\text { 5.3.3 Execução da AM no SUT } & 76\end{array}$

6. Experimentação do processo proposto 78

6.1 Funcionalidades testadas e estatísticas 78

6.2 Análise dos resultados 93

6.2.1 Avaliação quanto à eficiência 93

6.2.2 Avaliação quanto à eficácia 94

6.2.3 Evidências quanto às ferramentas Capture/Replay 97

$\begin{array}{ll}6.3 \text { Considerações } & 98\end{array}$

7. Conclusão 100

7.1 Limitações deste trabalho 103

7.2 Contribuições 104

7.3 Trabalhos futuros 104

8. Referências Bibliográficas 107 